\title{
Post Cataract Surgery Endophthalmitis Caused by Acinetobacter Baumannii
}

\author{
Shilpi H. Narnaware ${ }^{1}$, Prashant K. Bawankule ${ }^{2}$ \\ ${ }^{1}$ Fellowship in Vitreo-Retina, Consultant Vitreo-retina and ROP Specialist, Sarakshi Netralaya, 19, Rajiv Nagar, Wardha Road, \\ Nagpur-440025 Maharashtra, ${ }^{2}$ Vitreo-Retinal Surgeon, Sarakshi Netralaya, 19, Rajiv Nagar, Wardha Road, Nagpur-440025 \\ Maharashtra, India
}

Corresponding author: Dr. Shilpi H. Narnaware, Sarakshi Netralaya, Plot No. 19, Rajiv Nagar, Wardha Road, Nagpur-440025, Maharashtra, India

DOI: http://dx.doi.org/10.21276/ijcmsr.2019.4.3.8

How to cite this article: Shilpi H. Narnaware, Prashant K. Bawankule. Post cataract surgery endophthalmitis caused by acinetobacter baumannii. International Journal of Contemporary Medicine Surgery and Radiology. 2019;4(3):C38-C39.

\section{A B S T R A C T}

Introduction: Endophthalmitis caused by Acinetobacter Baumannii is emerging cause of infection resulting in poor visual outcomes.

Case report: We report a case of post cataract surgery endophthalmitis, who presented on second post- operative day. Aqueous tap was sent followed by intra-vitreal antibiotics on day of presentation. Following culture and sensitivity report systemic and intra-vitreal antibiotics were changed to match the spectrum of sensitivity. Patient recovered after 3 doses of intra-vitreal antibiotics on alternate day to 20/60 vision at glass appointment. Previous reports show poorer outcome in almost all the cases, except one, which showed improvement after vitrectomy. Our case was essentially managed only medically.

Conclusion: Timely diagnosis and prompt management according to drug sensitivity can prevent the devastating complications related to A.Baumannii infection.

Keywords: Acinetobacter Baumannii, Endophthalmitis.

\section{INTRODUCTION}

Endophthalmitis caused by Acinetobacter Baumannii is a rare but emerging cause of infection with poor visual and anatomical outcomes. There are reports of endogenous or post traumatic endophthalmitis with this bacteria. Few reports of post cataract endophthalmitis by A.Baumannii are reported in literature, indicating poorer visual and anatomical outcomes in maximum cases, despite intensive management. ${ }^{1-4}$ It is a slow growing gram negative bacterium which is generally an inhabitant of soil, vegetation and aquatic environment. It has emerged as one of the most troublesome pathogens for health care professionals because of its resistance to multiple drugs, as reported in various case series.

\section{CASE REPORT}

We report a case of acute post operative endophthalmitis caused by A Baumannii following uneventful cataract surgery. 70 year old, healthy, non- immunocompromised female, underwent right eye cataract surgery for senile cataract. On $2^{\text {nd }}$ post operative day, patient presented with complaints of blurred vision, redness and pain. Visual acuity was 3/60 on snellens chart. Examination revealed blood clot with streak hypopyon $(1 \mathrm{~mm})$ in anterior chamber (Fig 1). Fundus examination revealed vitreous haze of $3+$, with hazy view of disc. Ultrasound examination of right eye revealed moderate vitreous echoes (Fig 2) with attached retina with no evidence of thickening of coats. Aqueous tap was sent for culture and sensitivity and intravitreally Vancomycin (1 $\mathrm{mg} / 0.1 \mathrm{ml})$ \& Amikacin $(0.1 \mathrm{mg} / 0.1 \mathrm{ml})$ was given. On first follow up, anterior chamber hypopyon and vitreous haze persisted. Culture report revealed growth of Acinetobacter Baumanni and sensitivity report showed resistance to fluoroquinolones group / Gentamycin / Amikacin. Bacteria was found to be sensitive to Ceftazidime, Doxycyclin,

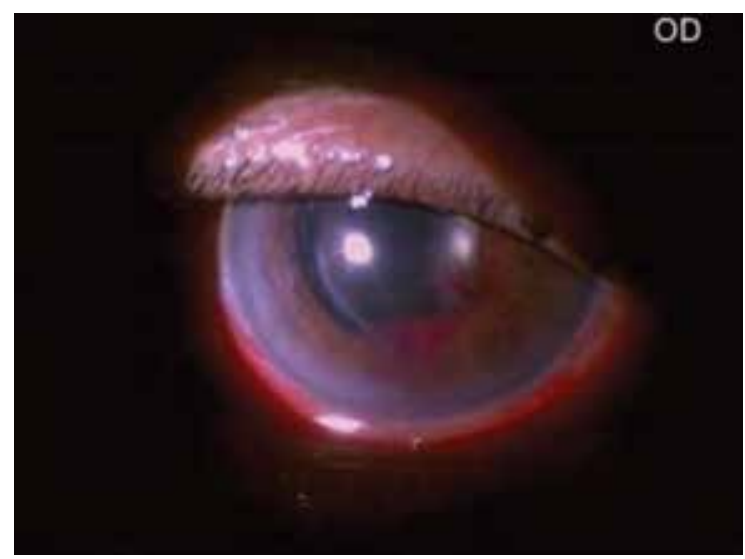

Figure-1: Slit Lamp Examination showing blood clot with streak hypopyon $(1 \mathrm{~mm})$ in anterior chamber 


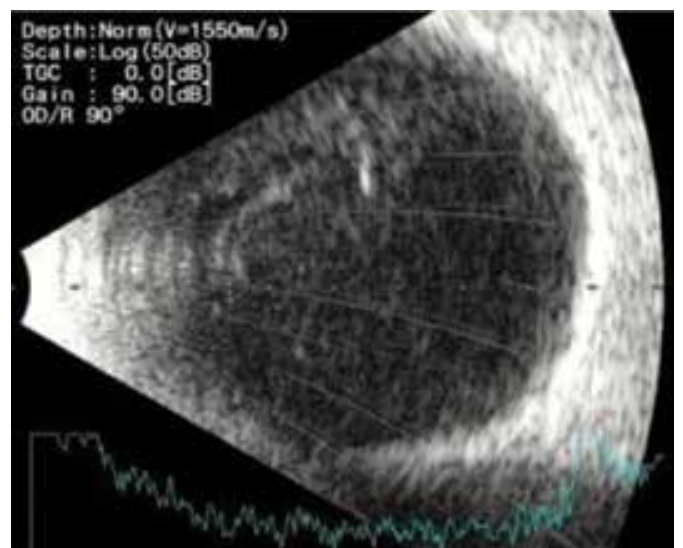

Figure-2: B-Scan revealing moderate vitreous echoes with attached retina

Colistin, Impenem, Sulbactum. Accordingly patient was started on oral Doxycycline $100 \mathrm{mg}$ twice a day for 10 days and intravitreally Vancomycin $(1 \mathrm{mg} / 0.1 \mathrm{ml})$, Ceftazidime $(2.25 \mathrm{mg} / 0.1 \mathrm{ml})$ and Dexamethasone $(0.4 \mathrm{mg} / 0.1 \mathrm{ml})$ on alternate day regimen. Patient received total 3 doses of intra-vitreal antibiotics. At final follow up best corrected visual acuity was 20/60, no hypopyon in anterior chamber, clear vitreous with healthy and full view of attached retina on fundus examination.

\section{DISCUSSION}

A.baumannii belongs to Moraxcellae family ${ }^{1}$ which can survive under a wide range of environmental conditions and persists on surfaces for extended periods. Recently, it has emerged as an important cause community acquired infections and a rare cause of endophthalmitis. Infection with A. Baumannii is an important cause of nosocomial infections. Chen et $\mathrm{al}^{2}$ has reported two cases, one was endogenous and other was post keratoplasty with outcome of 20/60 in 1 case and No PL in other. While out of four cases reported by Roy $\mathrm{R}$ et $\mathrm{al}^{3}$ three were post cataract surgery and one post trauma and they could salvage only one eye with visual outcome of 20/200. Favourable outcome after good microbiological evaluation and timely performed vitrectomy was reported by $\mathrm{G}$. Biigren et $\mathrm{al}^{4}$ in their case report. In our case we could manage the case medically with visual outcome of 20/60. Acquired resistance to multiple antibiotics and poor anatomical and visual prognosis poses a great challenge in front of surgeons regarding its management of infection by A.Baumannii. High level of sensitivity to Ciprofloxacin was reported by Gopal et $\mathrm{al}^{5}$ in another sub-species of acinetobacter, while Chen et $\mathrm{al}^{2}$ reported sensitivity to Imipenem in their series. Roy $\mathrm{R}$ et $\mathrm{al}^{3}$ reported $100 \%$ sensitivity to Ciprofloxacin and $100 \%$ resistance to Ceftazidime. In our case the bacteria was resistant to Ciprofloxacin and other fluoroquinolones while highly sensitive to Colistin, Ceftazidime and Doxycycline.

\section{CONCLUSION}

Antimicrobial resistance greatly limits the therapeutic options for patients who are infected with these bacteria. Timely diagnosis and prompt management according to drug sensitivity can prevent the devastating complications related to A.Baumannii infection. We were lucky to manage this case medically only and could salvage the eye.

\author{
Abbreviations \\ A.baumannii (Acinetobacter baumannii, $\mathrm{mg}$ (milligram), $\mathrm{ml}$ \\ (milli-litre), mm (milli-meter).
}

\section{REFERENCES}

1. Peleg Anton Y, S Harald, LP David. Acinetobacter baumannii: emergence of a successful pathogen. Clin Microbiol Rev 2008; 21 (3): 538-582

2. C Kuan-Jen, H Chiun-Ho, S Ming-Hui, L Chi-Chun, S Chi-Chin, H Ching-Hsi. Endophthalmitis caused by Acinetobacter baumannii: report of two cases. J Clin Microbiol. 2008;46(2):1148-1150.

3. R Roy, P Panigrahi, J Malathi, S S Pal, K Nandi, A Patil, E Nigam, and V Arora. Endophthalmitis caused by Acinetobacter baumanni: a case series. Eye (Lond). 2013; 27(3): 450-452.

4. GulfidanBitirgen,AhmetOzkagnici,Hurkan Kerimoglu, Umit Kamis. Acute postoperative endophthalmitis with an unusual infective agent: Acinetobacter baumannii. JCRS January 2013;39(1):143-144

5. Gopal,L.,A.A. Ramaswamy,H.Madhavan,M.Saswade, and R. R. Battu. 2000. Postoperative endophthalmitis caused by sequestered Acinetobacter calcoaceticus. Am. J. Ophthalmol. 129388-390.

Source of Support: Nil; Conflict of Interest: None

Submitted: 23-05-2019; Accepted: 30-06-2019; Published online: 24-07-2019 